NEURODEGENERATIVE DISEASE

\title{
Does tau pathology activate jumping genes?
}

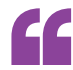 \\ tau-induced neurotoxicity was alleviated by therapeutic targeting of transposable element dysregulation}

Tau pathology - a hallmark of several neurodegenerative diseases might activate the movement of transposable elements, leading to genetic instability and neuronal death, according to two new studies. This mechanism could be a target for novel therapies for tauopathies.

Transposable elements - also known as jumping genes - are genetic sequences that can leap from one location to another in a genome. Although these elements form a large portion of the human genome, cells limit jumping through several epigenetic defences that include transcriptional silencing, via heterochromatin condensation, and post-transcriptional clearance, via PIWI-interacting RNAs (piRNAs). However, genomic instability increases in neurons in old age and in neurodegenerative disease. This change might partially result from a deterioration of epigenetic defences.

"I previously found that pathological forms of tau induce relaxation of constitutive heterochromatin," comments Bess Frost, corresponding author of one of the new reports.

"I was really quite keen to determine whether heterochromatin relaxation affected transposable

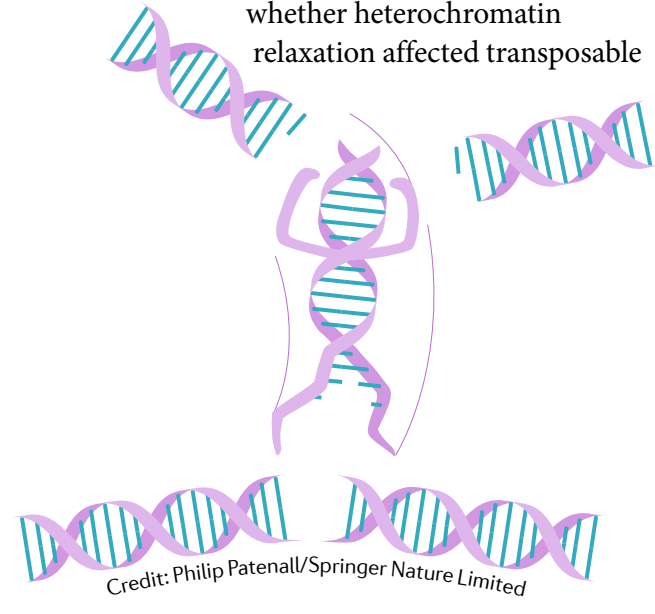

elements. When I began my own lab, we jumped right into the project."

Frost and colleagues used genetically modified Drosophila melanogaster to determine how tau affects jumping genes. The team found that expression of human tau that contained a disease-associated mutation substantially altered the expression of over 100 transposable elements in flies. Heterochromatin silencing and piRNA expression were both disrupted in tau-expressing flies. Furthermore, expression of mutant flamenco, which enhances transposable element mobility, dramatically increased neuronal death and reduced locomotor activity in flies expressing mutant tau, but not in control flies. These findings suggest that tau promotes neurodegeneration by disrupting transcriptional element silencing.

Promisingly, tau-induced neurotoxicity was alleviated by therapeutic targeting of transposable element dysregulation. Dietary restriction, which is known to extend lifespan in many species, reduced transposable element mobilization and ameliorated neuronal death and locomotor deficits in tau-expressing flies. Treatment with 3TC, a reverse transcriptase inhibitor currently approved by the FDA for treatment of HIV and hepatitis B, also had the same benefits.

Finally, post-mortem examination of brains from individuals who had Alzheimer disease $(\mathrm{AD})$, progressive supranuclear palsy or no neurodegeneration revealed that levels of the endogenous retrovirus class of transposable elements were higher in brains from patients with tauopathy than in brains from controls. "While we don't yet know if transposable elements are mobilizing in human tauopathy, these data suggest that the first step in retrotransposon mobilization transcription to RNA - occurs in human tauopathy," concludes Frost.

In a second study, Joshua Shulman and colleagues investigated how tau pathology burden correlates with dysregulation of transposable elements. "We wanted to examine whether we could find evidence of transposable element activity in association with $\mathrm{AD}$ pathological changes in human brains," explains Shulman.

The team carried out RNA sequencing in a series of 636 post-mortem brains from individuals with $\mathrm{AD}$, mild cognitive impairment or normal cognition. Neurofibrillary tangle burden correlated with greater activation of the endogenous retrovirus and LINE1 classes of transposable elements. Furthermore, chromatin relaxation was observed at $H E R V-F c 1$, one of the transposable elements upregulated in association with tau pathology.

Shulman and colleagues also examined transposable elements in Drosophila that express a human tau transgene. Expression of transposable elements was higher in tau-expressing flies than in wildtype controls, and this increased expression worsened with age.

"In my view, the critical next step is to determine whether transposable element activity truly mediates tau-induced neuronal injury or represents a non-causal biomarker," Shulman remarks. Research has also shown that gene jumping can activate the innate immune response, and Shulman and Frost both suggest that the potential role of tau-activated transposable elements in neuroinflammation presents an exciting avenue for future research.

Charlotte Ridler

ORIGINAL ARTICLES Sun, W. et al. Pathogenic tau-induced piRNA depletion promotes neuronal death through transposable element dysregulation in neurodegenerative tauopathies. Nat. Neurosci. 21, 1038-1048 (2018) | Guo, C. et al. Tau activates transposable elements in Alzheimer's disease. Cell Rep. 23, 2874-2880 (2018) 